\title{
Structure and band gaps of Ga-(V) semiconductors: The challenge of Ga pseudopotentials
}

\author{
O. Anatole von Lilienfeld and Peter A. Schultz* \\ Multiscale Dynamic Materials Modeling Department, Sandia National Laboratories, Albuquerque, New Mexico 87185-1322, USA
}

(Received 20 September 2007; published 10 March 2008)

\begin{abstract}
Design of gallium pseudopotentials has been investigated for use in density functional calculations of zinc-blende-type cubic phases of $\mathrm{GaAs}, \mathrm{GaP}$, and $\mathrm{GaN}$. A converged construction with respect to all-electron results is described. Computed lattice constants, bulk moduli, and band gaps vary significantly depending on pseudopotential construction or exchange-correlation functional. The Kohn-Sham band gap of the Ga-(V) semiconductors exhibits a distinctive and strong sensitivity to lattice constant, with near-linear dependence of gap on lattice constant for larger lattice constants and a $\Gamma-X$ crossover that changes the slope of the dependence. This crossover occurs at $\approx 98,101$, and $95 \%$ deviation from the equilibrium lattice constant for GaAs, $\mathrm{GaP}$, and GaN, respectively.
\end{abstract}

DOI: 10.1103/PhysRevB.77.115202

PACS number(s): 71.15.Mb, 71.20.Nr, 71.15.Dx, 31.30.-i

\section{INTRODUCTION}

The industrial importance of Ga-(V) semiconductors motivated many experimental and theoretical studies. ${ }^{1-3}$ In order to predict in an unbiased way the response of such materials to doping, defects, aging, engineering, extreme conditions, etc., an accurate $a b$ initio description is required. Firstprinciples calculations based on the Kohn-Sham (KS) density functional theory ${ }^{4,5}$ (DFT) are effective methods to study semiconductor properties. The fidelity of the exchangecorrelation functional fundamentally limits the accuracy of these methods in predicting materials properties. ${ }^{6}$ In addition, in most DFT methods for periodic systems, pseudopotentials replace explicit treatment of the core electrons, ${ }^{7-12}$ and the quality of the pseudopotentials can also significantly impact predicted properties. ${ }^{6}$ The tension in pseudopotential (PP) construction between computational convenience and robust transferability is particularly acute in gallium, where the $3 d^{10}$ shell is computationally demanding to treat explicitly as valence, but is also exceedingly difficult to model effectively as core electrons in a PP. We investigate the construction of PP for Ga to identify a converged PP design, and discuss the consequences of different compromises in the PP construction for bulk properties such as structure, bulk modulus, and, especially, the KS band gap.

In addition to emulating the effect of core electrons, a pseudopotential can be manipulated to approximately incorporate physical effects neglected in the functional, e.g., relativistic effects, self-interaction corrections, ${ }^{13,14}$ exact-exchange, ${ }^{15}$ or even van der Waals interactions ${ }^{16}$ and surface adsorption. ${ }^{17}$ Modifying the PP to obtain a widened KS band gap ${ }^{18,19}$ has also been proposed. The "band-gap" problem in KS-DFT is in fact not a failure of DFT as KSDFT is strictly a ground-state theory; only the electron density and total energy (and properties directly derived from these) are physically meaningful. A physically rigorous interpretation of other KS properties is still part of ongoing research. $^{20-22}$ The KS eigenfunctions and eigenenergies, however, are auxiliary functions of the KS equation and not electrons, and "must not be interpreted as corresponding to elementary excitations." 23 See Refs. 24 and 25 for a more rigorous (and accurate) theoretical treatment of excitation properties, such as the band gap, in GaN. Nevertheless, the size of the KS band gap, while formally not a meaningful predictor of the experimental band gap, ${ }^{23}$ is a crucial quantity for practical DFT-based studies aiming at predicting defects in materials. The narrowing of the KS-DFT gap with respect to experiment ${ }^{26,27}$ may cause desired defect states to be buried within the conduction or valence band, preventing construction of a valid computational model of a defect state. In fact, it was this observation that prompted this study: using an "optimal" PP within a generalized gradient approximated density functional, the predicted band gap for GaAs was $\approx 0.1 \mathrm{eV}$, rendering it unsuitable for investigation of defects in GaAs.

For this study, our desire is to fashion a PP that retains integrity for structural energetics, yet permits meaningful defect studies in the future. We systematically explore the effect of the Ga-pseudopotential generation on the prediction of structure and band gaps in Ga-(V) semiconductors. For the KS exchange-correlation potential, we used both the local density approximation (LDA) ${ }^{28}$ with the fit of Perdew and Zunger ${ }^{29}$ and the generalized gradient approximation (GGA) of Perdew, Burke, and Ernzerhof (PBE), ${ }^{30}$ and screen a variety of plausible $\mathrm{PP}$ approximations for $\mathrm{Ga}$ in zincblende-type crystals of GaAs, GaP, and GaN. The importance of the details in the pseudopotential and exchangecorrelation approximation in GaN studies has been discussed for more than a decade, ${ }^{31-33}$ and the literature is abundant, see Ref. 34 (and references therein). For GaAs, however, the effect of semicore orbitals on self-energy corrections was studied only recently within the GW approximation and a plane-wave basis. ${ }^{35}$ Our emphasis is on the adequacy of different PP for DFT based studies on GaAs, but we also examine $\mathrm{GaP}$ and $\mathrm{GaN}$ to gauge the broader transferability of the different PP constructions for gallium. For this study, different pseudopotential formulations, different numbers of valence electrons, different oxidation states of the all electron reference atom, as well as scalar relativistic and nonrelativistic cores have been explored by computing equilibrium lattice constants, bulk moduli, and the band gap dependency on volume and pressure. The exploration of the band gap behavior appears to reveal a universal direct-to-indirect crossing as a function of lattice constant, near the equilibrium volume. 


\section{METHODOLOGY}

\section{A. Construction of pseudopotentials}

For all atoms standard norm-conserving pseudopotentials have been chosen, in what is conventionally referred to as the "semilocal" form, i.e., without the additional approximation of the Kleinman-Bylander ${ }^{36}$ separation. The construction of ultra-soft pseudopotentials, ${ }^{37}$ without the norm-conserving constraint, would require a different prescription for developing transferable pseudopotentials than described here. However, once such an ultrasoft PP (or any different PP) is converged to a transferable form, it should, in principle, exhibit the same behavior in use as a transferable normconserving PP. While the specific PP construction might be different, many of the issues we discuss should be general, and the subsequent predicted properties of the materials, e.g., band gaps and lattice parameters, should be the same, i.e., transferable.

For the nitrogen atom, a Troullier-Martins ${ }^{38}$ pseudopotential was constructed, treating the $1 s^{2}$ shell as core electrons and the $2 s^{2} 2 p^{3}$ electrons as valence. The "core radius" of the PP core, the $R_{c}$, for both the $s$ and $p$ potential was $1.25 \mathrm{Bohr}$, and the $l=1$ potential was used as the local potential. In tests of small molecule properties, this construction was converged with respect to a (much more computationally expensive) Hamann pseudopotential. ${ }^{39}$

The arsenic and phosphorus pseudopotentials were generated using the Hamann ${ }^{39}$ construction, with the Hamann $\operatorname{program}^{39}$ for the LDA and the FHI98PP program ${ }^{40}$ for all PBE atoms. Both used a five-electron $s^{2} p^{3}$ valence configuration, and default $R_{c}$ for the $s$ and $p$ valence angular momentum channels. We refer to Refs. 39 and 40 for details regarding these defaults. The $R_{c}$ for the arsenic $d$ potential was set to the default (1.87 Bohr for LDA, 1.83 for PBE), while the default $R_{c}$ for phosphorus was $1.08 \mathrm{Bohr}$ (both LDA and PBE). This concluded the PP for phosphorus, as the resulting $l=2$ potential was adequately hard to be used as an accurate local potential.

For a standard arsenic PP, denoted As' in the following, the PP also concluded with the $l=2$ potential, and used the $l=2$ as the local potential. We also constructed an alternative, harder, "extended" PP for arsenic, prompted by the observation that the $3 d^{10}$ core shell is rather shallow (arsenic being only two atoms over from gallium in the Periodic Table), and hence the resulting $l=2$ valence PP is rather soft. In this second arsenic, we extended the As PP to $l=3$, setting its $R_{c}=1.2 \mathrm{Bohr}$ to make it very hard and thus making it better than the $l=2$ for the local potential. Further, we added a nonlinear core correction $^{41}$ with a matching radius of 1.6 Bohr, to include the pertinent core density in the construction of the valence exchange-correlation potential.

For the gallium atom, we explored a variety of different PP constructions, as listed in Table I. For consistency, the Fritz-Haber Institute FHI98PP code $^{40}$ was used for the generation of all Ga pseudopotentials, both PBE and LDA. The PP were constructed independently for LDA and PBE, and as another means to manipulate the effective potential, the $\mathrm{Ga}$ atom was treated as either nonrelativistic (n) and scalar relativistic (s). We note that the valence orbital eigenstates of the
TABLE I. Employed functionals and Ga pseudopotentials. The nomenclature is explained as follows: first nonrelativistic (n) or scalar relativistic $(\mathrm{s})$, then neutral $\left({ }^{0}\right)$ or cationic $\left({ }^{+}\right)$all electron reference atom, then Hamann $(\mathrm{H})$ or Troullier $(\mathrm{T})$ for the $s$ and $p$ channels, then $13(d)$ valence electrons or 3 [blank, and only for (H)].

\begin{tabular}{cccccccccc}
\hline \hline & 1 & 2 & 3 & 4 & 5 & 6 & 7 & 8 & 9 \\
\hline LDA & $\mathrm{s}^{0} \mathrm{H} d$ & $\mathrm{~s}^{0} \mathrm{~T} d$ & $\mathrm{~s}^{0} \mathrm{H}$ & $\mathrm{s}^{+} \mathrm{H} d$ & $\mathrm{~s}^{+} \mathrm{T} d$ & $\mathrm{~s}^{+} \mathrm{H}$ & $\mathrm{n}^{+} \mathrm{H} d$ & $\mathrm{n}^{+} \mathrm{T} d$ & $\mathrm{n}^{+} \mathrm{H}$ \\
PBE & & & & $\mathrm{s}^{+} \mathrm{H} d$ & $\mathrm{~s}^{+} \mathrm{T} d$ & $\mathrm{~s}^{+} \mathrm{H}$ & $\mathrm{n}^{+} \mathrm{H} d$ & $\mathrm{n}^{+} \mathrm{T} d$ & $\mathrm{n}^{+} \mathrm{H}$ \\
\hline \hline
\end{tabular}

PP are low enough in energy to be minimally affected by scalar relativistic effects directly. The principal effect of relativistic effects is in the inner core region, ${ }^{39}$ and the impact on the valence states is mostly to respond to a relativistically shrunken core. There is no spin-orbit in the DFT calculation, and the atom PP is averaged over the spin-orbit term, so that consistency between atomic PP and valence DFT is not significantly violated with or without scalar relativistic PP.

The number of valence electrons was taken to be either three (blank designation), with the $3 d^{10}$ shell in the core, or thirteen (designated with $d$ ), with the $3 d^{10}$ electrons in the valence.

The $3 d$-core PP (valence charge three) was constructed using the Hamann method for all channels, with a default $R_{c}$ for the $d$ potential (2.05 Bohr for all cases), and adding a hard $f$ potential, $R_{c}=1.2 \mathrm{Bohr}$, to use as the local potential. A nonlinear core correction ${ }^{41}$ with a $1.6 \mathrm{Bohr}$ matching radius was also added to this PP. For the $3 d^{10}$-valence PP (valence charge thirteen), the $l=2$ channel was constructed using the Troullier-Martins method with $R_{c}=1.5 \mathrm{Bohr}$, and this $l=2$ potential was used as the local potential, terminating this PP. The Hamann $d$ potential was too hard to be convenient for our application, and this TM $d$ potential has been shown to be converged for bulk properties ${ }^{6}$ of $3 d$ transition metals.

The Ga PP was constructed from either a neutral $\left({ }^{0}\right)$, $\left(d^{10}\right) s^{2.0} p^{1.0}$, or a cation $\left(^{+}\right),\left(d^{10}\right) s^{1.5} p^{0.5}$, atomic valence configuration. Using a positively charged atomic configuration has been seen to give better PP for weakly bound states in metallic atoms, including $3 d$ transition metal atoms. ${ }^{6}$ In all cases, the $R_{c}$ for the Ga $s$ and $p$ potentials were shortened from the default values to improve the potentials, the $R_{c}$ for the final $s$-potential ranging from 1.01 to $1.09 \mathrm{Bohr}(\mathrm{H})$, and 1.73 to $1.77 \mathrm{Bohr}(\mathrm{T})$, and the $p$-potential ranging from 1.04 to $1.14 \mathrm{Bohr}(\mathrm{H})$, and set to $2.05 \mathrm{Bohr}(\mathrm{T})$. Consistency for results between $(\mathrm{H})$ - and $(\mathrm{T})$-based potentials verified this construction (and verification served as the principal motivation for this apparent duplication), just as the neutral atomic configuration PP was done to verify that nothing was amiss with the cation PP.

\section{B. Computational procedures}

All calculations were performed with SEQQUEST, ${ }^{42}$ a fully self-consistent Gaussian-based linear combination of atomic orbitals code. A well-optimized valence double-zeta plus polarization basis set was used on all atoms. The Ga-(V) zincblende bulk crystal calculations were performed using eight- 
atom cubic cells, with $52^{3}$ and $56^{3}$ real space integration grids for LDA and PBE, respectively. A $6^{3}$ regular $k$-space grid centered at the $\Gamma$ point was used to sample reciprocal space. The ground-state structure of GaN is wurtzite, but we have chosen to examine the zinc-blende structure for this study as it facilitates comparisons with results from $\mathrm{GaP}$ and GaAs, both of which are zinc-blende crystals. Total energy, lattice constant, and bulk modulus have been converged with respect to real space grid and $k$-point grid. The $3 d$-valence $\mathrm{PP}$ converges more slowly with the grid than the $3 d$-core PP, but all properties are well modeled using a $6^{3} k$ grid with the eight-atom cubic unit cell.

The bulk moduli and lattice constants were obtained according to Birch ${ }^{43}$ and Murnaghan ${ }^{44}$ fitting a third order polynomial to energies at lattice constants spaced in regular increments of $0.5 \%$ within at least $-3 /+3$ and $-3 /+5 \%$ of the experimental lattice constant for LDA and PBE, respectively.

The $k$ space was centered at $\Gamma$ rather than offset from $\Gamma$ to specifically include the valence band maximum and, hence, the direct KS band gap, in the sampling space of the groundstate calculation. The conduction band minimum in the following was taken from the minimum conduction band eigenvalue within the $k$ sample. While not exact in identifying the crossover from direct to indirect, we assume that the $k$ sample is dense enough that this is sufficient for our purposes.

\section{RESULTS AND DISCUSSION}

The formally "best" $\mathrm{Ga}$ pseudopotentials we constructed $-d^{10}$-valence, scalar-relativistic, cation, Hamann $s$ and $p\left(\mathrm{~s}^{+} \mathrm{H} d\right)$-in concert with the best (extended PP) arsenic yields a lattice parameter of $5.63 \AA$ and bulk modulus of $73 \mathrm{GPa}$ with the LDA functional, and $5.77 \AA$ and $61 \mathrm{GPa}$ with the PBE functional. This is in nearly perfect agreement with the corresponding all-electron values ${ }^{45} 5.62 \AA$ and $72 \mathrm{GPa}$ (LDA) or $5.76 \AA$ and $60 \mathrm{GPA}$ (PBE), obtained using a full-potential linear muffin tin orbital (FP-LMTO) code, ${ }^{46,47}$ or with the LDA FP-LMTO results of Fiorentini et al. ${ }^{31} 5.62 \AA$ and $75 \mathrm{GPa}$, respectively. But also for GaP and $\mathrm{GaN}$, the agreement with all-electron results is very satisfying. The GaN LDA benchmark PP lattice constant of $4.50 \AA$ compares well with the lattice constants from all-electron calculations, ranging between 4.44 and $4.47 \AA .{ }^{31,48,49}$ The LDA bulk modulus of $194 \mathrm{GPa}$ compares relatively well with all-electron results ranging from 198 to $208 \mathrm{GPa} .{ }^{31,48,49}$ This indicates that the design of these PP is sound, and that these benchmark PP should be effective for modeling GaAs bulk ground-state properties.

The difficulty with using the benchmark PP for GaAs lies in the resulting KS band gaps. The KS band gap for these benchmark PP in the LDA at the theoretical lattice parameter is $0.42 \mathrm{eV}$. Although much smaller than the experimental $(0 \mathrm{~K})$ band gap of $1.52 \mathrm{eV},{ }^{50}$ this is roughly comparable to the DFT band gap in Si where such a KS band gap proved adequate to accurately predict a wide spectrum of defect levels ${ }^{51}$ with an average error of $0.1 \mathrm{eV}$ throughout the full width of the experimental band gap. This LDA band gap for
GaAs, while perhaps marginal, could prove adequate for bulk defect studies in GaAs. However, the KS band gap for PBE with these benchmark PP is $\approx 0.1 \mathrm{eV}$, rendering them unusable for meaningful defect calculations (except for special cases). Our desire is to identify a PP that retains integrity for structural energetics, yet permits meaningful defect studies. We begin with considering alternative PP constructions, and their performance for structural properties, as a starting point for examining the behavior of the KS band gaps.

\section{A. Lattice constants and bulk moduli}

Table II displays the computed lattice constants and bulk moduli for Ga-(V) zinc-blende structures, using the benchmark Ga PP and all its variants. In comparison to experiment, the LDA lattice constants using the benchmark PP are very good, less than $1 \%$ too small for GaAs(-0.4\%) and $\mathrm{GaP}(-0.6 \%)$, and exact for zinc-blende GaN. The LDA bulk moduli are also very good, within less than $10 \%$ for all three compounds. The PBE comparisons are not as good, being $1.5-2.0 \%$ too large in the lattice parameter, and up to $23 \%$ too small for the bulk modulus. Nonetheless, these are within the typical accuracy obtained with DFT, and the smaller lattice constants and larger bulk moduli of LDA vs. PBE are also typical of bulk results comparing these two functionals. Overall, the LDA does significantly better than GGA-PBE for the structural properties of $\mathrm{Ga}-(\mathrm{V})$ compounds.

As noted above, the benchmark PP almost exactly reproduces the all-electron results, confirming the desired convergence of the PP. Results using the optimized (T) and (H) $s$ and $p$ potentials are essentially identical, additional evidence that this part of the PP is converged. The cation atom PP and the neutral atom PP also give negligibly different results, demonstrating that the PP is robust to significant changes in its construction. The only (quite modest) exception is that the $3 d$-core potential results for the cation $\mathrm{s}^{+} \mathrm{H}$ are slightly improved over the neutral $\mathrm{s}^{0} \mathrm{H}$. This last could be expected because this $d$ potential in the $3 d$-core PP is very weak, and, as we noted previously, a cation tends to give better PP for weakly bound states in metals, and, here, it appears also to give an improved PP for a weakly bound state in a semiconductor. A non-relativistic Ga atom PP results in a lattice parameter larger by $\approx 0.01 \AA$, and also a slightly larger bulk modulus (by less than $2 \mathrm{GPa}$ ). This is not a large effect, to be expected for an atom this early in the Periodic Table.

The effect of accounting for the $3 d$ electrons in the core is much greater. For computational efficiency, using a $3 d$-core potential for Ga would be preferred over a $3 d$-valance PP, but the results in Table II suggest caution in making this substitution. Although not as severe as in $\mathrm{GaN}$, where it has been demonstrated that a $3 d$-valence Ga potential is needed to get good results,${ }^{32}$ the differences between results with the $3 d$-core and $3 d$-valence potentials in GaAs and GaP are significant, particularly for the lattice parameter.

The use of the extended As PP-nonlinear core correction and hard local potential - causes the GaAs lattice constant to decrease by $\approx 0.02 \AA$ with the Ga $3 d$-valence PP, accompanied by a slight decrease in the bulk modulus. With a $3 d$-core $\mathrm{PP}$ on the Ga, there is no meaningful difference. This impli- 
TABLE II. Calculated zinc-blende-type lattice constants $(\AA)$ and bulk moduli $(\mathrm{GPa})$ for zinc-blende-type Ga-(V) crystals. GaAs' and GaAs correspond to the $\operatorname{As}(l=2)$ and $\operatorname{As}(l=3)$ pseudopotential, respectively. The PP column is explained in Table I. "Other" refers to all-electron results in the literature.

\begin{tabular}{|c|c|c|c|c|c|c|c|c|}
\hline PP & \multicolumn{4}{|c|}{ lattice constants } & GaAs' & \multicolumn{2}{|c|}{ bulk moduli } & $\mathrm{GaN}$ \\
\hline LA & & & & & & & & \\
\hline${ }^{0} \mathrm{H} d$ & 5.647 & 5.628 & 5.420 & 4.500 & 74.3 & 72.6 & 86.9 & 195.2 \\
\hline $\mathrm{s}^{0} \mathrm{~T} d$ & 5.647 & 5.628 & 5.419 & 4.499 & 74.3 & 72.6 & 87.0 & 195.3 \\
\hline $\mathrm{s}^{0} \mathrm{H}$ & 5.587 & 5.586 & 5.384 & 4.380 & 73.1 & 73.2 & 88.9 & 199.8 \\
\hline${ }^{+} \mathrm{H} d$ & 5.647 & 5.628 & 5.419 & 4.498 & 74.2 & 72.6 & 86.3 & 194.0 \\
\hline $\mathrm{s}^{+} \mathrm{T} d$ & 5.646 & 5.627 & 5.418 & 4.497 & 74.4 & 72.8 & 86.5 & 194.3 \\
\hline $\mathrm{s}^{+} \mathrm{H}$ & 5.599 & 5.599 & 5.397 & 4.392 & 72.5 & 72.4 & 87.8 & 200.0 \\
\hline $\mathrm{n}^{+} \mathrm{H} d$ & 5.659 & 5.641 & 5.437 & 4.510 & 75.3 & 73.6 & 86.9 & 194.3 \\
\hline $\mathrm{n}^{+} \mathrm{T} d$ & 5.658 & 5.641 & 5.436 & 4.508 & 75.5 & 73.7 & 87.0 & 194.6 \\
\hline$n^{+} \mathrm{H}$ & 5.612 & 5.613 & 5.413 & 4.408 & 73.3 & 73.2 & 88.4 & 199.5 \\
\hline Other ${ }^{\mathrm{a}}$ & \multicolumn{2}{|c|}{5.62} & 5.40 & 4.47 & \multicolumn{2}{|c|}{72} & 89 & 199 \\
\hline Other ${ }^{b}$ & \multicolumn{2}{|c|}{5.62} & & 4.47 & \multicolumn{3}{|c|}{75} & 198 \\
\hline Other ${ }^{\mathrm{c}}$ & \multirow{2}{*}{\multicolumn{3}{|c|}{$5.62^{d}$}} & 4.46 & & & & 200 \\
\hline Other & & & & $4.44^{\mathrm{e}}$ & \multicolumn{3}{|c|}{$74^{\mathrm{d}}$} & $205^{\mathrm{e}}$ \\
\hline \multicolumn{9}{|l|}{ PBE } \\
\hline $\mathrm{s}^{+} \mathrm{H} d$ & 5.793 & 5.767 & 5.532 & 4.590 & 60.5 & 59.8 & 75.4 & 164.4 \\
\hline $\mathrm{s}^{+} \mathrm{T} d$ & 5.794 & 5.768 & 5.532 & 4.591 & 60.5 & 59.8 & 75.4 & 164.3 \\
\hline $\mathrm{s}^{+} \mathrm{H}$ & 5.749 & 5.739 & 5.506 & 4.484 & 59.1 & 59.6 & 75.7 & 169.2 \\
\hline $\mathrm{n}^{+} \mathrm{H} d$ & 5.804 & 5.780 & 5.549 & 4.602 & 61.6 & 60.9 & 76.5 & 165.2 \\
\hline $\mathrm{n}^{+} \mathrm{T} d$ & 5.802 & 5.778 & 5.547 & 4.600 & 62.0 & 61.2 & 76.7 & 165.7 \\
\hline $\mathrm{n}^{+} \mathrm{H}$ & 5.762 & 5.753 & 5.523 & 4.501 & 60.3 & 60.9 & 76.4 & 169.3 \\
\hline Other ${ }^{\mathrm{a}}$ & \multirow{2}{*}{\multicolumn{2}{|c|}{5.76}} & 5.52 & 4.55 & \multirow{2}{*}{\multicolumn{2}{|c|}{60}} & 76 & 171 \\
\hline Other ${ }^{\mathrm{c}}$ & & & & 4.55 & & & & 172 \\
\hline Exp. ${ }^{f}$ & \multicolumn{2}{|c|}{5.65} & 5.45 & 4.50 & \multicolumn{2}{|c|}{79} & 88 & 190 \\
\hline
\end{tabular}

${ }^{\mathrm{a}}$ Full-potential linear muffin-tin orbitals (FP-LMTO) results from Ref. 45.

bFP-LMTO results from Ref. 31.

${ }^{\mathrm{c}}$ Full-potential linear augmented plane wave (FP-LAPW) results from Ref. 52.

${ }^{\mathrm{d}}$ Linear augmented plane wave (LAPW) results from Ref. 53.

eFP-LAPW results from Ref. 49.

fLattice constants: GaAs, Ref. 1; GaP, Ref. 50; GaN, Ref. 2; Bulk moduli: GaAs, Extrapolation to $T \approx 0 \mathrm{~K}$, Ref. 1 ; GaP, Ref. 54; GaN, Ref. 55.

cates a residual $3 d-3 d$ interaction between the As and $\mathrm{Ga}$. In a final analysis, the extended As PP, with respect to the standard As PP, improves the treatment of GaAs, but only slightly, and could perhaps be safely omitted for computationally more extensive studies of GaAs.

\section{B. Band gaps}

Compared with the computed structural properties of Ga(V) compounds, the KS band gap is much more sensitive to PP construction and exchange-correlation functional. The
TABLE III. Calculated zinc-blende-type KS band gaps $E_{g}(\mathrm{eV})$, for $\mathrm{Ga}-(\mathrm{V})$ crystals at the theoretical $a_{0}^{t}$ and experimental lattice constants $a_{0}^{e}$. GaAs' and GaAs correspond to the $\operatorname{As}(l=2)$ and $\operatorname{As}(l=3)$ pseudopotential, respectively. The PP column is explained in Table I.

\begin{tabular}{|c|c|c|c|c|c|c|c|c|}
\hline \multirow[b]{2}{*}{ PP } & \multicolumn{2}{|c|}{ GaAs' } & \multicolumn{2}{|c|}{ GaAs } & \multicolumn{2}{|c|}{$\mathrm{GaP}$} & \multicolumn{2}{|c|}{$\mathrm{GaN}$} \\
\hline & $E_{g}\left(a_{0}^{e}\right)$ & $E_{g}\left(a_{0}^{t}\right)$ & $E_{g}\left(a_{0}^{e}\right)$ & $E_{g}\left(a_{0}^{t}\right)$ & $E_{g}\left(a_{0}^{e}\right)$ & $E_{g}\left(a_{0}^{t}\right)$ & $E_{g}\left(a_{0}^{e}\right)$ & $E_{g}\left(a_{0}^{t}\right)$ \\
\hline \multicolumn{9}{|l|}{ LDA } \\
\hline $\mathrm{s}^{0} \mathrm{H} d$ & 0.34 & 0.35 & 0.32 & 0.41 & 1.48 & 1.46 & 1.81 & 1.90 \\
\hline $\mathrm{s}^{0} \mathrm{~T} d$ & 0.35 & 0.37 & 0.33 & 0.42 & 1.49 & 1.46 & 1.82 & 1.92 \\
\hline $\mathrm{s}^{0} \mathrm{H}$ & 0.51 & 0.79 & 0.49 & 0.77 & 1.55 & 1.49 & 2.15 & 2.93 \\
\hline $\mathrm{s}^{+} \mathrm{H} d$ & 0.41 & 0.42 & 0.38 & 0.47 & 1.49 & 1.46 & 1.88 & 1.98 \\
\hline $\mathrm{s}^{+} \mathrm{T} d$ & 0.43 & 0.45 & 0.41 & 0.50 & 1.49 & 1.46 & 1.91 & 2.02 \\
\hline $\mathrm{s}^{+} \mathrm{H}$ & 0.63 & 0.86 & 0.61 & 0.83 & 1.56 & 1.52 & 2.27 & 2.99 \\
\hline $\mathrm{n}^{+} \mathrm{H} d$ & 0.68 & 0.64 & 0.65 & 0.69 & 1.53 & 1.51 & 2.04 & 2.09 \\
\hline $\mathrm{n}^{+} \mathrm{T} d$ & 0.71 & 0.67 & 0.68 & 0.72 & 1.53 & 1.51 & 2.08 & 2.13 \\
\hline $\mathrm{n}^{+} \mathrm{H}$ & 0.88 & 1.05 & 0.87 & 1.02 & 1.59 & 1.55 & 2.41 & 3.02 \\
\hline Other & & & $0.29^{\mathrm{a}}$ & & & $1.42^{\mathrm{b}}$ & $2.1 / 1$ & $9^{\mathrm{c}}, 2.1^{\mathrm{d}}$ \\
\hline \multicolumn{9}{|l|}{ PBE } \\
\hline $\mathrm{s}^{+} \mathrm{H} d$ & 0.59 & 0.03 & 0.60 & 0.13 & 1.63 & 1.53 & 1.81 & 1.50 \\
\hline $\mathrm{s}^{+} \mathrm{T} d$ & 0.63 & 0.06 & 0.64 & 0.17 & 1.63 & 1.56 & 1.85 & 1.53 \\
\hline $\mathrm{s}^{+} \mathrm{H}$ & 0.82 & 0.40 & 0.83 & 0.45 & 1.70 & 1.74 & 2.20 & 2.40 \\
\hline $\mathrm{n}^{+} \mathrm{H} d$ & 0.88 & 0.27 & 0.89 & 0.37 & 1.66 & 1.70 & 1.99 & 1.63 \\
\hline $\mathrm{n}^{+} \mathrm{T} d$ & 0.98 & 0.37 & 0.98 & 0.47 & 1.66 & 1.72 & 2.09 & 1.73 \\
\hline $\mathrm{n}^{+} \mathrm{H}$ & 1.09 & 0.62 & 1.10 & 0.67 & 1.72 & 1.79 & 2.36 & 2.46 \\
\hline Other & & & $0.40^{\mathrm{e}}$ & & & & $1.75^{f}$ & $1.83^{\mathrm{g}}$ \\
\hline Exp. ${ }^{\mathrm{h}}$ & \multicolumn{4}{|c|}{1.4} & \multicolumn{2}{|c|}{2.35} & \multicolumn{2}{|c|}{3.2} \\
\hline
\end{tabular}

ainear augmented plane wave (LAPW) results at $a_{0}^{e}$ from Ref. 53. ${ }^{\mathrm{b}}$ At $a_{0}^{t}$ with Ga $d$ electrons in the PP core (Ref. 56).

${ }^{\mathrm{c}}$ At $a_{0}^{e}$ with Ga $d$ electrons in the PP core/valence, respectively (Ref. 34).

${ }^{\mathrm{d}}$ At $a_{0}^{e}$ with Ga $d$ electrons in the PP core with partial core correction (Ref. 24), a GW approximation in the same study predicts $3.1 \mathrm{eV}$.

${ }^{\mathrm{e}} \mathrm{GGA}$ (PW91) LAPW results at $a_{0}^{e}$ from Ref. 53.

${ }^{\mathrm{f}}$ Full potential (FP) LAPW PBE results at $a_{0}^{e}$ from Ref. 57.

gFP-LAPW PBE results at $a_{0}^{t}$ from Ref. 58.

${ }^{\mathrm{h}} \mathrm{GaAs}$ : Ref. 59; GaP: Ref. 50; GaN: Ref. 2.

computed KS band gaps for $\mathrm{Ga}-(\mathrm{V})$ zinc-blende structure compounds using variants of Ga PP is presented in Table III, at both the experimental $\left(a_{0}^{e}\right)$ and appropriate theoretical $\left(a_{0}^{t}\right)$ lattice parameters. Although the structural properties of Ga(V) materials vary by only a few percent, the KS band gaps exhibit qualitative differences with change in PP and functional. For example, at the theoretical lattice parameter, the PBE KS band gap for $\mathrm{Ga}\left(\mathrm{s}^{+} \mathrm{H} d\right) \mathrm{As}^{\prime}$ is only $0.03 \mathrm{eV}$, while the $\mathrm{Ga}\left(\mathrm{s}^{+} \mathrm{H}\right) \mathrm{As}^{\prime}$ produces a $0.40 \mathrm{eV}$ band gap. But also for LDA the KS band gap can vary from $0.35 \mathrm{eV}$ for $\mathrm{Ga}\left(\mathrm{s}^{0} \mathrm{H} d\right) \mathrm{As}^{\prime}$ up to $1.05 \mathrm{eV}$ for the nonrelativistic $3 d$-core $\mathrm{PP}, \mathrm{Ga}\left(\mathrm{n}^{+} \mathrm{H}\right) \mathrm{As}^{\prime}$. The differences are proportionately less in $\mathrm{GaP}$ and GaN, but the $3 d$-core and $3 d$-valence PP result in KS band gaps that differ by as much as $1 \mathrm{eV}$ in $\mathrm{GaN}$, too. The extended As PP causes the KS band gap to slightly de- 

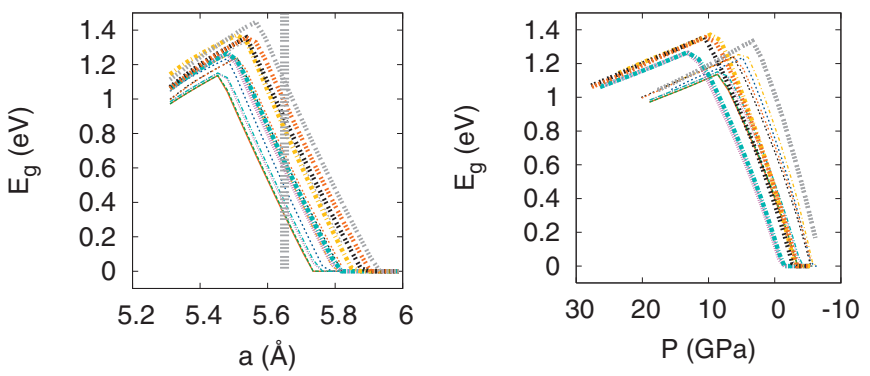

\begin{tabular}{|c|c|c|}
\hline 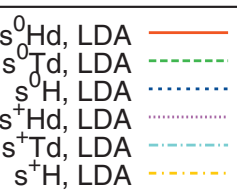 & 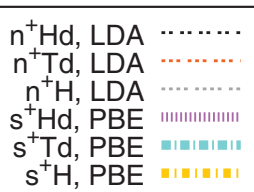 & 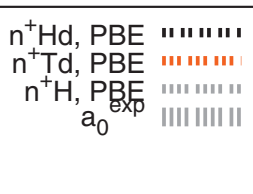 \\
\hline
\end{tabular}

FIG. 1. (Color) GaAs LDA and PBE KS band gaps $E_{g}(\mathrm{eV})$ versus lattice constant $a(\AA)$ (left) and versus pressure $P(\mathrm{GPa})$ (right) for various Ga pseudopotentials and using the As pseudopotential. Details on the pseudopotentials are given in Table I and in the methodological section.

crease at $a_{0}^{e}$, but then changes to a slight increase at $a_{0}^{t}$ for the $\mathrm{Ga} 3 d$-valence PP. From these results it becomes evident that before any of these PP can be used in predictive simulations, the origins of this large variability in such a central computational observable as the KS band gap needs to be understood.

Band gap dependence. Particularly for GaAs and GaN, and less so for $\mathrm{GaP}$, the KS band gap is significantly different in going from the theoretical lattice constant $a_{0}^{t}$ to the experimental lattice constant $a_{0}^{e}$. This suggests a strong dependence of the KS band gap on the lattice constant, as was also noted for GaN. ${ }^{25}$ To investigate the details of this dependence, we computed the KS band gap as a function of lattice constant or pressure and display the results for GaAs in Fig. 1, for $\mathrm{GaP}$ in Fig. 2, and for $\mathrm{GaN}$ in Fig. 3. In these plots, the $\mathrm{KS}$ band gap does indeed vary greatly depending on the lattice constant. Moreover, the shape of that dependence is common to all the Ga-(V) compounds. For larger lattice constants, the KS band gap of GaAs is zero. When decreasing the lattice constant towards the equilibrium lattice constant, the direct gap rises steeply as the $a_{0}$ gets smaller, and nearly linearly with $a_{0}$, until a crossover at $\approx 98,101$, and $95 \%$ of the experimental lattice constant for $\mathrm{GaAs}, \mathrm{GaP}$, and $\mathrm{GaN}$, respectively. At that point, an off- $\Gamma$ state crosses below the conduction band minimum resulting in a band gap transition changing from direct $(\Gamma \rightarrow \Gamma)$ to indirect $(\Gamma \rightarrow X)$. If one associates the band gap to the electronic hardness of atoms and molecules, ${ }^{60}$ this maximum in the vicinity of the equilibrium lattice constant is reminiscent of the maximum hardness principle, as suggested by Pearson ${ }^{61,62}$ and later on proved by Parr and Chattaraj. ${ }^{63}$

GaAs. The direct-indirect crossover has been observed before theoretically in GaAs. Theoretical predictions of its location vary from $3.05 \mathrm{GPa}$ by Christensen ${ }^{18}$ to $8 \mathrm{GPa}$ by Vast and co-workers. ${ }^{64}$ Our results for GaAs indicate the transition to be between $3 \mathrm{GPa}$ and $13 \mathrm{GPa}$ depending on the $\mathrm{Ga} \mathrm{PP}$ and functional, and being at $\approx 6 \mathrm{GPa}$ using the LDA
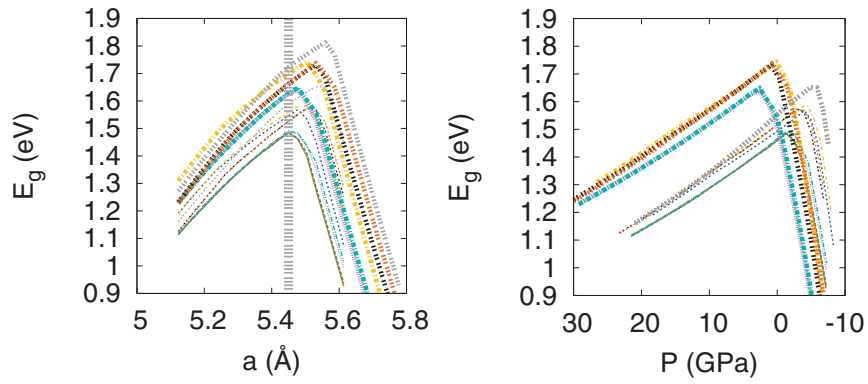

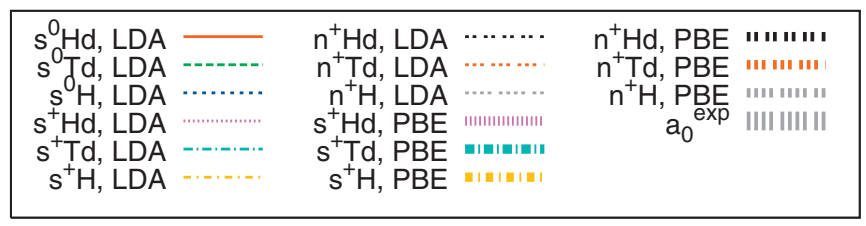

FIG. 2. (Color) GaP LDA and PBE KS band gaps $E_{g}(\mathrm{eV})$ versus lattice constant $a(\AA)$ (left) and versus pressure $P(\mathrm{GPa})$ (right) for various Ga pseudopotentials. Details on the pseudopotentials are given in Table I and in the methodological section.

benchmark Ga PP and $\approx 13 \mathrm{GPa}$ using the PBE benchmark Ga PP. Goñi et al. ${ }^{59}$ presented experimental data with the crossover at $4.2 \pm 0.2 \mathrm{GPa}$ (and $\approx 1.9 \mathrm{eV}$ ). The computed DFT transition pressure compares well with this, and the LDA results agree better with experiment than the PBE results.

$G a P$. Experimentally, GaP has an indirect band gap, consistent with the current LDA results that indicate a negative pressure would be required to observe a transition to a direct band gap. The PBE band gap for GaP, in contrast, is direct at the theoretical lattice constant with all the PP we consideronce again, LDA appears to give superior results to PBE.

$G a N$. The GaN band gap is direct for all PP and functionals, consistent with experiment, and our prediction indicates that the zinc-blende crystal would need to be at a very high pressure to observe a transition to an indirect band gap.
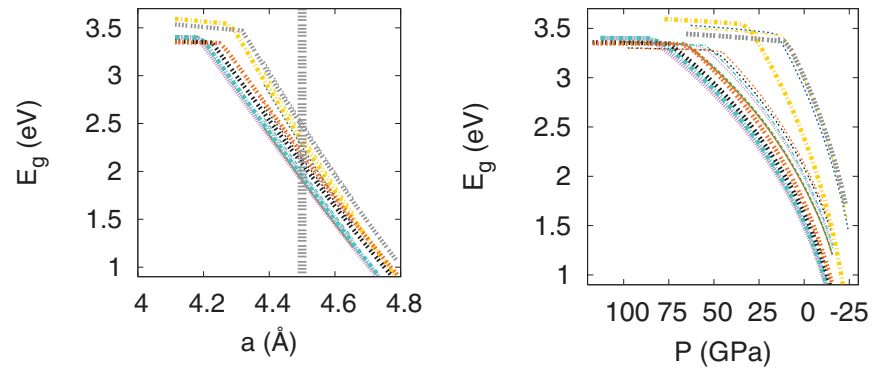

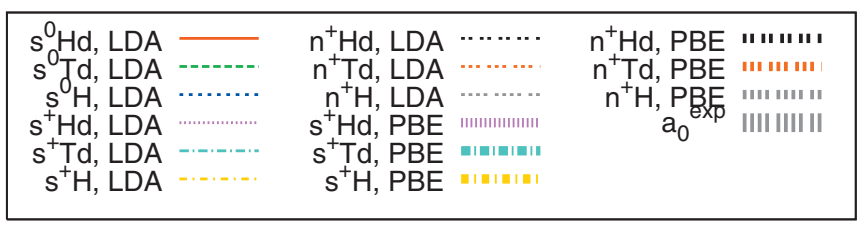

FIG. 3. (Color) GaN LDA and PBE KS band gaps $E_{g}(\mathrm{eV})$ versus lattice constant $a(\AA)$ (left) and versus pressure $P(\mathrm{GPa})$ (right) for various Ga pseudopotentials. Details on the pseudopotentials are given in Table I and in the methodological section. 
While we note again that the KS eigenvalue band gap is not predictive of a band gap excitation energy, these results indicate that, just as for the structural properties, the LDA seems to do qualitatively better than GGA-PBE for Ga-(V) compounds in describing electronic properties such as the band gap dependence on the lattice constant. The linear behavior of the KS band gap around the zero pressure in Figs. $1-3$ is in qualitative agreement with the pressure dependence published in Ref. 3. Comparison of numerical values of pressure coefficients to experiments or literature does not appear to be straightforward as these coefficients depend strongly on the kind of fit (linear or sublinear) and the investigated pressure range, as pointed out by Dunstan and co-workers. ${ }^{65}$ As such, a detailed discussion of the "deformation potential" of the band gap with lattice constant, volume, or pressure, goes beyond the scope of this work. Despite the fact that the use of different PP and functionals introduces significant variance in the numerical predictions for the band gap at a given lattice constant, the shapes of the lattice constant vs band gap curve are very similar. For example, the slope of the direct band gap vs lattice constant is nearly the same for all the PP and functionals. The choice of PP and functional appears merely to shift the lattice constant where the transition occurs, and its height. Plotting gap vs pressure, the GGA results clearly separate from LDA results, with the transition occurring at higher pressures for LDA. For the GaP, as noted above, this results in the PBE predicting a direct band gap transition, except for the $n^{+} \mathrm{H}-\mathrm{PP}$, rather than the indirect transition observed in experiment.

Effect of lattice constant. Notably for GaAs, but also for $\mathrm{GaN}$, the KS band gap at the theoretical lattice constant is smaller with PBE than it is with LDA, with a nearly zero KS band gap for the benchmark PP in PBE. However, at the experimental lattice constant, the PBE yields a larger KS band gap than LDA for all compounds. This is consistent with the all electron LDA and PW91 results for GaAs, ${ }^{53}$ see Table III. For most PP, the PBE yields a larger peak band gap at the indirect-direct transition than the LDA. Hence, the expression of a smaller theoretical band gap for PBE can be explained by the steep dependence of the band gap on the lattice constant and the fact that PBE generally predicts larger lattice constants. The KS band gap of GaP does not exhibit the same band gap variability with lattice constant as seen in $\mathrm{GaAs}$ and $\mathrm{GaN}$, because for $\mathrm{GaP}$ both the experimental and the theoretical lattice constants sit at or near the direct-indirect transition rather than on the steep slope of the direct band gap. The $3 d$-core Ga PP exhibit larger band gaps than $3 d$-valence PP. This is partly due to electronic effects stemming from $3 d$-valence effects, but in GaAs and $\mathrm{GaN}$ the lattice size effects dominate as this modest increase in gap is strongly magnified when the band gap descends the steep slope vs lattice constant as the crystal is relaxed to the PBE theoretical lattice constant.

The extended As PP causes little change in the KS band gap, comparing results fixed at the experimental lattice constant, but more significant changes at the theoretical lattice constant. This again can be correlated with the band gap dependence in lattice constant. The explicit presence of $3 d$ electrons in the valence causes the KS band gap to decrease, but, again, the dominant effect is due to a structural relax- ation and the strong dependence of the band gap on the lattice constant.

We have also examined the effect of using nonrelativistic $\mathrm{Ga}$ atoms to construct the Ga PP. This early in the Periodic Table, however, the effect of scalar relativity in the atoms should be minor. The results for structural properties in Table II and for the KS band gaps in Table III bear this out. As expected, the nonrelativistic lattice constants are larger, by just slightly more than $0.01 \AA$. A little more surprising, the bulk modulus also becomes larger (although not by much) even though a bulk modulus typically, but not necessarily, decreases as the lattice constant increases. Finally, the computed KS band gap is larger for a nonrelativistic Ga atom than for the corresponding scalar-relativistic PP, by as much as $\approx 0.2 \mathrm{eV}$ for the GaAs. This runs counter to the trend observed within the scalar-relativistic Ga PP of a larger lattice parameter yielding a smaller band gap. This indicates that the band gap dependency on the lattice constant can be compensated by electronic effects. With the nonrelativistic $\mathrm{Ga}$ atom in Ga-(V) compounds, the semicore $3 d$-shell will be deeper relative to the valence $s$ and $p$ shells, leading to larger band gaps than for the relativistic $\mathrm{Ga}$. The consequence is that one could resort to a nonrelativistic $\mathrm{Ga}$ atom to help expand the band gap in GaAs, without a significant impact on the structural energetics.

\section{CONCLUSIONS}

We have examined the structural and KS band gap properties of zinc-blende structure Ga-(V) compounds with pseudopotential methods, systematically investigating the effects of different plausible PP constructions and functionals on computed properties. We outlined a prescription for generating a Ga pseudopotential that accurately reproduces results of corresponding full-potential all-electron results, that requires placing the semicore $3 d$ electrons in the valence. The choice of PP construction can significantly alter the DFT predictions, particularly for the KS band gaps, with the most significant differences being between $3 d$-core and $3 d$-valence PP.

The band gap dependency on the volume in the Ga-(V) compounds follows a characteristic pattern, with a directindirect transition in the band gap near the equilibrium volume. Of particular concern for computational studies is the steep dependence of the band gap on lattice constant for the direct gap (GaAs and GaN) compounds. Predictions of band gaps in $\mathrm{Ga}-(\mathrm{V})$, even if the KS eigenvalues did correspond to excitation energies, would be problematic due to a high sensitivity of the KS band gap on functional, pseudopotential, and, especially, the corresponding predicted lattice constant. One severe consequence is that the GGA-PBE KS band gap of GaAs approaches zero as the theoretical equilibrium lattice constant is overestimated, making realistic calculations of defect properties in GaAs with PBE problematic. However, the variability with PP can be exploited to expand the KS band gap without strongly compromising structural properties, and thereby perhaps allow meaningful defect studies. Using an extended As PP, or using a nonrelativistic Ga atom both lead to slight expansions of the KS band gap. A $3 d$-core 
PP has the biggest benefit to the band gap, but the history in $\mathrm{GaN}$, and our results above for the structural properties of Ga-(V) suggest that this substitution should be done very carefully and skeptically.

We expect the various atomic PP described here to be rather transferable between different possible III-V alloys, allowing for electronic structure studies of ternary alloys such as $\mathrm{GaAs}_{1-x} \mathrm{~N}_{x}{ }^{66-69}$ We note, however, that only the PP for $\mathrm{Ga}$ in $\mathrm{Ga}-(\mathrm{V})$ have been tested here, and it may well be the case that for a description of interactions involving other atoms than $\mathrm{Ga}$, such as a As-N interaction for example, it may prove necessary to promote the As $3 d$ into the valence in order to get the best results.

Finally, it is interesting to note that despite the fact that PBE is a GGA approximation and nominally "better" than LDA, LDA proves universally better than PBE for predicting crystal properties of these Ga-(V) compounds. The LDA lattice constants and bulk moduli all are good predictions of experimental behavior, unlike the PBE, and, due to the strong band gap dependency, also the LDA band gap prop- erties are qualitatively better than PBE. It is noteworthy, however, that when imposing the same lattice constant PBE does predict a larger band gap than LDA. This points out the hazards of tuning new functionals to artificially increase the KS band gap: the resulting change in structure can induce a larger change in the KS band gap than the direct electronic effect from changing the functional. Whether LDA will prove better when applied to the chemistry of defects in Ga(V) remains an open question.

\section{ACKNOWLEDGMENTS}

We are grateful for comments and structural all-electron results for GaAs, GaP, and GaN provided by Ann E. Mattsson. ${ }^{45}$ OAvL acknowledges support from SNL Truman Program LDRD Project No. 120209. Sandia is a multiprogram laboratory operated by Sandia Corporation, a Lockheed Martin Company, for the United States Department of Energy's National Nuclear Security Administration under Contract No. DE-AC04-94AL85000. *paschul@sandia.gov

${ }^{1}$ J. S. Blakemore, J. Appl. Phys. 53, R123 (1982).

${ }^{2}$ T. Lei, T. D. Moustakas, R. J. Graham, Y. He, and S. J. Berkowitz, J. Appl. Phys. 71, 4933 (1992).

${ }^{3}$ S. Q. Wang and H. Q. Ye, J. Phys.: Condens. Matter 14, 9579 (2002).

${ }^{4}$ P. Hohenberg and W. Kohn, Phys. Rev. 136, B864 (1964).

${ }^{5}$ W. Kohn and L. J. Sham, Phys. Rev. 140, A1133 (1965).

${ }^{6}$ A. Mattsson, P. Schultz, M. Desjarlais, T. Mattsson, and K. Leung, Modell. Simul. Mater. Sci. Eng. 13, R1 (2005).

${ }^{7}$ H. Hellmann, J. Chem. Phys. 3, 61 (1935).

${ }^{8}$ H. Hellmann, J. Chem. Phys. 4, 324 (1936).

${ }^{9}$ J. C. Phillips and L. Kleinman, Phys. Rev. 116, 287 (1959).

${ }^{10}$ J. D. Weeks and S. A. Rice, J. Chem. Phys. 49, 2741 (1968).

${ }^{11}$ G. B. Bachelet, D. R. Hamann, and M. Schluter, Phys. Rev. B 26, 4199 (1982).

${ }^{12}$ P. A. Christiansen, Y. S. Lee, and K. S. Pitzer, J. Chem. Phys. 71, 4445 (1979).

${ }^{13}$ M. M. Rieger and P. Vogl, Phys. Rev. B 52, 16567 (1995).

${ }^{14}$ B. Baumeier, P. Krüger, and J. Pollmann, Phys. Rev. B 73, 195205 (2006).

${ }^{15}$ O. A. von Lilienfeld, I. Tavernelli, U. Rothlisberger, and D. Sebastiani, J. Chem. Phys. 122, 014113 (2005).

${ }^{16}$ O. A. von Lilienfeld, I. Tavernelli, U. Rothlisberger, and D. Sebastiani, Phys. Rev. Lett. 93, 153004 (2004).

${ }^{17}$ A. Tkatchenko and O. A. von Lilienfeld, Phys. Rev. B 73, 153406 (2006).

${ }^{18}$ N. E. Christensen, Phys. Rev. B 30, 5753 (1984).

${ }^{19}$ D. Segev, A. Janotti, and C. G. Van de Walle, Phys. Rev. B 75, 035201 (2007).

${ }^{20}$ E. J. Baerends and O. V. Gritsenko, J. Phys. Chem. A 101, 5383 (1997).

${ }^{21}$ Ralf Stowasser and Roald Hoffmann, J. Am. Chem. Soc. 121, 3414 (1999).

${ }^{22}$ J. F. Janak, Phys. Rev. B 18, 7165 (1978).
${ }^{23}$ L. J. Sham and W. Kohn, Phys. Rev. 145, 561 (1966).

${ }^{24}$ A. Rubio, J. L. Corkill, M. L. Cohen, E. L. Shirley, and S. G. Louie, Phys. Rev. B 48, 11810 (1993).

${ }^{25}$ M. Palummo, L. Reining, R. W. Godby, C. M. Bertoni, and N. Börnsen, Europhys. Lett. 26, 607 (1994).

${ }^{26}$ L. J. Sham and M. Schlüter, Phys. Rev. Lett. 51, 1888 (1983).

${ }^{27}$ L. J. Sham and M. Schlüter, Phys. Rev. B 32, 3883 (1985).

${ }^{28}$ D. M. Ceperley and B. J. Alder, Phys. Rev. Lett. 45, 566 (1980).

${ }^{29}$ J. P. Perdew and A. Zunger, Phys. Rev. B 23, 5048 (1981).

${ }^{30}$ J. P. Perdew, K. Burke, and M. Ernzerhof, Phys. Rev. Lett. 77, 3865 (1996).

${ }^{31}$ V. Fiorentini, M. Methfessel, and M. Scheffler, Phys. Rev. B 47, 13353 (1993).

${ }^{32}$ A. F. Wright and J. S. Nelson, Phys. Rev. B 50, 2159 (1994).

${ }^{33}$ C. Stampfl and C. G. Van de Walle, Phys. Rev. B 59, 5521 (1999).

${ }^{34}$ B. Bouhafs, F. Litimein, Z. Dridi, and P. Ruterana, Phys. Status Solidi B 236, 61 (2003).

${ }^{35}$ M. L. Tiago, S. Ismail-Beigi, and S. G. Louie, Phys. Rev. B 69, 125212 (2004).

${ }^{36}$ L. Kleinman and D. M. Bylander, Phys. Rev. Lett. 48, 1425 (1982).

${ }^{37}$ D. Vanderbilt, Phys. Rev. B 41, 7892 (1990).

${ }^{38}$ N. Troullier and J. L. Martins, Phys. Rev. B 43, 8861 (1991).

${ }^{39}$ D. R. Hamann, Phys. Rev. B 40, 2980 (1989).

${ }^{40}$ M. Fuchs and M. Scheffler, Comput. Phys. Commun. 119, 67 (1999).

${ }^{41}$ S. G. Louie, S. Froyen, and M. L. Cohen, Phys. Rev. B 26, 1738 (1982).

${ }^{42} \mathrm{http} / / / \mathrm{dft}$. sandia.gov/Quest/

${ }^{43}$ F. Birch, Phys. Rev. 71, 809 (1947).

${ }^{44}$ F. D. Murnaghan, Am. J. Math. 49, 235 (1937).

${ }^{45}$ A. E. Mattsson and T. R. Mattsson (unpublished).

${ }^{46}$ J. M. Wills, O. Eriksson, M. Alouani, and D. L. Price, FullPotential LMTO Total Energy and Force Calculations, Vol. 535 
of Electronic Structure and Physical Properties of Solids-The Uses of the LMTO Method Lecture Notes in Physics, edited by Hugues Dreyssé (Springer, Berlin, 2000).

${ }^{47}$ Download from http://www.rspt.net/

${ }^{48}$ M. van Schilfgaarde, A. Sher, and A.-B. Chen, J. Cryst. Growth 178, 8 (1997).

${ }^{49}$ V. A. Gubanov, A. F. Wright, J. S. Nelson, C. Y. Fong, Z. W. Lu, B. M. Klein, and D. R. Hamann, Phys. Status Solidi B 209, 63 (1998).

${ }^{50}$ I. Vurgaftman, J. R. Meyer, and L. R. Ram-Mohan, J. Appl. Phys. 89, 5815 (2001).

${ }^{51}$ P. A. Schultz, Phys. Rev. Lett. 96, 246401 (2006).

${ }^{52}$ M. Fuchs, J. L. F. Da Silva, C. Stampfl, J. Neugebauer, and M. Scheffler, Phys. Rev. B 65, 245212 (2002).

${ }^{53}$ C. Filippi, D. J. Singh, and C. J. Umrigar, Phys. Rev. B 50, 14947 (1994).

${ }^{54}$ Group IV Elements, IV-IV and III-V Compounds, Part a-Lattice Properties, Landolt-Börnstein-Group III Condensed Matter (Springer Verlag, Berlin, 2001), Vol. 41A1a.

${ }^{55}$ M. E. Sherwin and T. J. Drummond, J. Appl. Phys. 69, 8423 (1991).

${ }^{56}$ A. Höglund, C. W. M. Castleton, and S. Mirbt, Phys. Rev. B 72, 195213 (2005).
${ }^{57}$ R. Ahmed, H. Akbarzadeh, and Fazal-e-Aleem, Physica B 370, 52 (2005)

${ }^{58}$ C. Persson, A. Ferreira da Silva, R. Ahuja, and B. Johansson, J. Cryst. Growth 231, 397 (2001).

${ }^{59}$ A. R. Goñi, K. Strössner, K. Syassen, and M. Cardona, Phys. Rev. B 36, 1581 (1987).

${ }^{60}$ P. Geerlings, F. D. Proft, and W. Langenaeker, Chem. Rev. (Washington, D.C.) 103, 1793 (2003).

${ }^{61}$ R. G. Pearson, J. Am. Chem. Soc. 85, 3533 (1963).

${ }^{62}$ R. G. Pearson, J. Chem. Educ. 64, 561 (1987).

${ }^{63}$ R. G. Parr and P. K. Chattaraj, J. Am. Chem. Soc. 113, 1854 (1991).

${ }^{64}$ J. Sjakste, V. Tyuterev, and N. Vast, Phys. Rev. B 74, 235216 (2006).

${ }^{65}$ M. D. Frogley, J. L. Sly, and D. J. Dunstan, Phys. Rev. B 58, 12579 (1998).

${ }^{66}$ L. Bellaiche, S.-H. Wei, and A. Zunger, Phys. Rev. B 54, 17568 (1996).

${ }^{67}$ P. R. C. Kent and A. Zunger, Phys. Rev. Lett. 86, 2613 (2001).

${ }^{68}$ E. Arola, J. Ojanen, H.-P. Komsa, and T. T. Rantala, Phys. Rev. B 72, 045222 (2005).

${ }^{69}$ K. Laaksonen, H.-P. Komsa, E. Arola, T. T. R., and R. M. Nieminen, J. Phys.: Condens. Matter 18, 10097 (2006). 